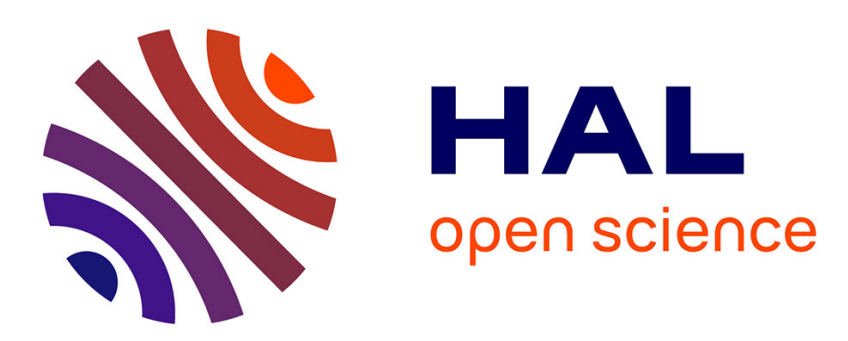

\title{
Le rooibos d'Afrique du Sud : comment la biodiversité s'invite dans la construction d'une indication géographique
}

\author{
Estelle Biénabe, Maya Leclercq, Pascale Moity-Maïzi
}

\section{To cite this version:}

Estelle Biénabe, Maya Leclercq, Pascale Moity-Maïzi. Le rooibos d'Afrique du Sud: comment la biodiversité s'invite dans la construction d'une indication géographique. Autrepart - Revue de sciences sociales au Sud, 2009, 10.3917/autr.050.0117 . hal-02959486

\author{
HAL Id: hal-02959486 \\ https://hal.science/hal-02959486
}

Submitted on 8 Oct 2020

HAL is a multi-disciplinary open access archive for the deposit and dissemination of scientific research documents, whether they are published or not. The documents may come from teaching and research institutions in France or abroad, or from public or private research centers.
L'archive ouverte pluridisciplinaire HAL, est destinée au dépôt et à la diffusion de documents scientifiques de niveau recherche, publiés ou non, émanant des établissements d'enseignement et de recherche français ou étrangers, des laboratoires publics ou privés. 


\title{
LE ROOIBOS D'AFRIQUE DU SUD : COMMENT LA BIODIVERSITÉ S'INVITE DANS LA CONSTRUCTION D'UNE INDICATION GÉOGRAPHIQUE
}

\author{
Estelle Biénabe, Maya Leclercq, Pascale Moity-Maïzi
}

Presses de Sciences Po | «Autrepart »

2009/2 $\mathrm{n}^{\circ} 50 \mid$ pages 117 à 134

ISSN 1278-3986

ISBN 9782724631654

Article disponible en ligne à l'adresse :

https://www.cairn.info/revue-autrepart-2009-2-page-117.htm

Distribution électronique Cairn.info pour Presses de Sciences Po.

(C) Presses de Sciences Po. Tous droits réservés pour tous pays.

La reproduction ou représentation de cet article, notamment par photocopie, n'est autorisée que dans les limites des conditions générales d'utilisation du site ou, le cas échéant, des conditions générales de la licence souscrite par votre établissement. Toute autre reproduction ou représentation, en tout ou partie, sous quelque forme et de quelque manière que ce soit, est interdite sauf accord préalable et écrit de l'éditeur, en dehors des cas prévus par la législation en vigueur en France. Il est précisé que son stockage dans une base de données est également interdit. 


\title{
Le rooibos d'Afrique du Sud : comment la biodiversité s'invite dans la construction d'une indication géographique
}

\author{
Estelle Biénabe*, Maya Leclercq**, Pascale Moity-Maïzi***
}

\section{Introduction}

Cet article s'inscrit dans un questionnement général sur le rôle que pourraient jouer les indications géographiques (IG) comme outils de conservation de la biodiversité. Les IG, selon la définition inscrite dans l'accord de l'OMC sur les aspects des droits de propriété intellectuelle qui touchent au commerce (ADPIC), « servent à identifier un produit comme étant originaire du territoire d'un membre, ou d'une région ou localité de ce territoire, dans les cas où une qualité, réputation ou autre caractéristique déterminée du produit peut être attribuée essentiellement à cette origine géographique » [art. $22: 1]$. À partir du cas du rooibos, tisane produite à partir d'une plante endémique à l'Afrique du Sud, nous nous proposons d'éclairer les interrogations que soulève la construction d'une IG dans un contexte spécifique, celui d'une filière en forte expansion, et de montrer comment la biodiversité intervient dans cette construction. Il est important de souligner le caractère émergent des IG en Afrique du Sud. Il n'existe pas de cadre légal et institutionnel spécifique pour leur mise en œuvre. Le processus de réflexion autour du potentiel de développement d'une IG pour le rooibos a été engagé en 2006 avec les acteurs de la filière. Cette démarche est actuellement la plus avancée en Afrique du Sud et permet d'alimenter une réflexion nationale sur les changements institutionnels à même de promouvoir le développement d'IG comme instruments de développement rural et de conservation de la biodiversité.

L'hypothèse principale sur laquelle s'appuie notre analyse est la suivante : les conditions dans lesquelles se déroule la construction d'une démarche de qualification d'une production localisée autour de l'IG contribuent à l'élaboration de cadres de référence collectifs permettant d'intégrer les enjeux de conservation de la biodiversité spécifique à un milieu. Le développement des IG s'opère en effet

* CIRAD - UMR Innovation, Université de Pretoria, bienabe@ cirad.fr

** Doctorante MNHN, CIRAD-UMR Innovation, Université de Cape Town, leclercq@mnhn.fr

*** Montpellier Supagro, UMR Innovation, pascale.maizi@supagro.inra.fr 
localement grâce à un processus d'adaptation des normes véhiculées par l'IG et de codification des pratiques. Il repose ainsi sur de multiples négociations et arbitrages, mettant en scène différents acteurs. Ce processus peut donc être l'occasion de créer de nouveaux espaces de médiation, utiles pour construire de nouvelles règles partagées autour de la gestion des ressources spécifiques engagées dans la production d'une IG. Pour cette analyse, nous nous appuyons à la fois sur des données d'entretiens réalisés avec des producteurs de rooibos sur toute la zone de production, dans le cadre du programme de recherche ANR Biodivalloc, et sur les observations tirées de différentes réunions organisées pour la mise en place du projet d'IG rooibos. Nos informations sont ainsi essentiellement produites à travers une démarche ethnographique privilégiant l'observation participante et la triangulation des informations [Olivier de Sardan, 1995].

Pour comprendre le contexte dans lequel se construit cette IG, nous caractérisons dans une première partie la trajectoire du rooibos, en précisant les différents éléments qui participent à en faire aujourd'hui un produit de terroir (endémicité, territorialité, histoire locale reconnue). Dans une deuxième partie, nous montrons comment l'irruption de nouvelles préoccupations associées à l'évolution des marchés et de la filière suscite l'émergence de nouveaux dispositifs (IG et biodiversité) qui sont porteurs de nouvelles perspectives de qualification collective des pratiques. Pour mieux saisir les enjeux associés à ces dispositifs, nous illustrons la grande diversité des pratiques, des ancrages dans le territoire et des points de vue autour de ces pratiques et de la qualité du rooibos, à travers deux trajectoires contrastées de valorisation individuelle du rooibos. Nous montrons alors dans une troisième partie comment la biodiversité, en «s'associant» au dispositif de qualification collective construit autour de l'IG, permet d'une part, de raisonner différentes formes de production à travers la codification de «bonnes pratiques », d'autre part de créer de nouveaux référents communs.

\section{Le Rooibos : un produit de terroir sud-africain}

Le rooibos, également connu en France sous le nom de thé rouge, est une plante endémique d'Afrique du Sud dont on produit une tisane de couleur rouge après fermentation ${ }^{1}$ des feuilles et de la partie supérieure des tiges. Il a probablement été découvert pour la première fois par les populations locales, les Khoekhoe ${ }^{2}$, au XVIII ${ }^{\mathrm{e}}$ ou XIX ${ }^{\mathrm{e}}$ siècle, même s'il n'existe pas de références historiques le confirmant ${ }^{3}$. C'est au cours du $\mathrm{XX}^{\mathrm{e}}$ siècle que sa production s'accroît; il devient un produit de consommation courante d'abord en Afrique du Sud puis en

\footnotetext{
1. Si cette opération est appelée communément fermentation, il s'agit en fait d'une oxydation enzymatique.

2. Les Khoekhoe sont connus sous le nom de «Hottentot» en français. Ils sont souvent désignés par le terme générique «Khoisan», qui regroupe les populations Khoekhoe (éleveurs-cueilleurs) et San (chasseur-cueilleurs).

3. Les Khoekhoe sont généralement cités comme les premiers utilisateurs du rooibos : ils connaîtraient ses vertus médicinales et l'auraient utilisé en tant que médecine [CAELERS, 2007]. Toutefois, il n'existe pas de trace écrite mentionnant l'usage du rooibos par les Khoekhoe.
} 
Occident où ses ventes atteignent $65 \%$ de la production totale en 2003 [Tisa, 2004].

\section{Caractéristiques du rooibos : origine, pratiques, réputation, territorialité}

Le rooibos, production inscrite dans une histoire relativement courte (documentée uniquement à partir de 1904), est doté de caractéristiques reconnues qui concourent à en faire un produit spécifique. Tout d'abord, l'origine de ce produit est double. L'héritage présumé khoekhoe du rooibos est globalement reconnu par les acteurs de la production, et son nom Afrikaans ${ }^{4}$ montre clairement le lien de ce produit avec la culture des fermiers sud-africains blancs qui sont à l'origine des processus de mise sur le marché et de mise en culture. Ces fermiers ont fait évoluer les techniques associées à la production (mécanisation par exemple). Malgré un développement technologique évident, les acteurs de la filière considèrent que la chaîne opératoire de production (antérieure à la stérilisation et à l'emballage) a peu évolué. Le rooibos est récolté pendant l'été puis transporté jusqu'au lieu de transformation, généralement situé sur la ferme, où il est coupé puis rassemblé en tas pour fermenter. Il est alternativement arrosé et compressé afin de libérer les tanins qui, en contact avec une enzyme oxydante, se transforment chimiquement et commencent à changer de couleur. Il s'agit d'un procédé clef, dont la maîtrise s'acquiert avec l'expérience. C'est dans cette transformation que le rooibos prend sa couleur caractéristique rouge-brun et son arôme spécifique [Ginsberg, 1976]. Le thé « rouge » est ensuite répandu en fines couches afin de sécher, puis il est transporté jusqu'à l'usine où il est conditionné pour la vente selon des procédés variables en fonction des commandes.

Au-delà de ses propriétés organoleptiques le rooibos est connu comme une tisane à vertus médicinales. Les recherches scientifiques démontrent aujourd'hui que le rooibos contient peu de tanins, pas de théine, différents types d'antioxydants (flavonoïdes, acides phénoliques, polyphénols...) recherchés pour leurs propriétés contre le vieillissement, et qu'il est probablement doté de propriétés anti-allergéniques [Erickson, 2003 ; South African Rooibos Council, 2008].

L'aire de distribution du rooibos est restreinte, localisée dans le sud-ouest de l'Afrique du Sud. Le rooibos appartient à un cortège floristique nommé fynbos, également localisé et possédant un taux élevé d'endémisme végétal, caractérisé notamment par la présence de protées. Si le rooibos spontané s'étend jusqu'à la péninsule du Cap, l'aire du rooibos cultivé s'inscrit dans un rayon de $100 \mathrm{~km}$ environ autour de Clanwilliam (carte 1). C'est à partir de cet espace de production que les acteurs locaux (producteurs et transformateurs) distinguent une aire de production «traditionnelle » et une aire dite d'expansion. La première s'étend à environ $50 \mathrm{~km}$ à l'ouest, à l'est et au sud et à $100 \mathrm{~km}$ au nord de Clanwilliam, région d'où provient également la variété de rooibos sauvage sélectionnée. À

4. Ce mot signifie littéralement «buisson rouge ». Il désigne à la fois la plante qui, en séchant, devient rouge et le produit fini, de couleur rouge après fermentation. 


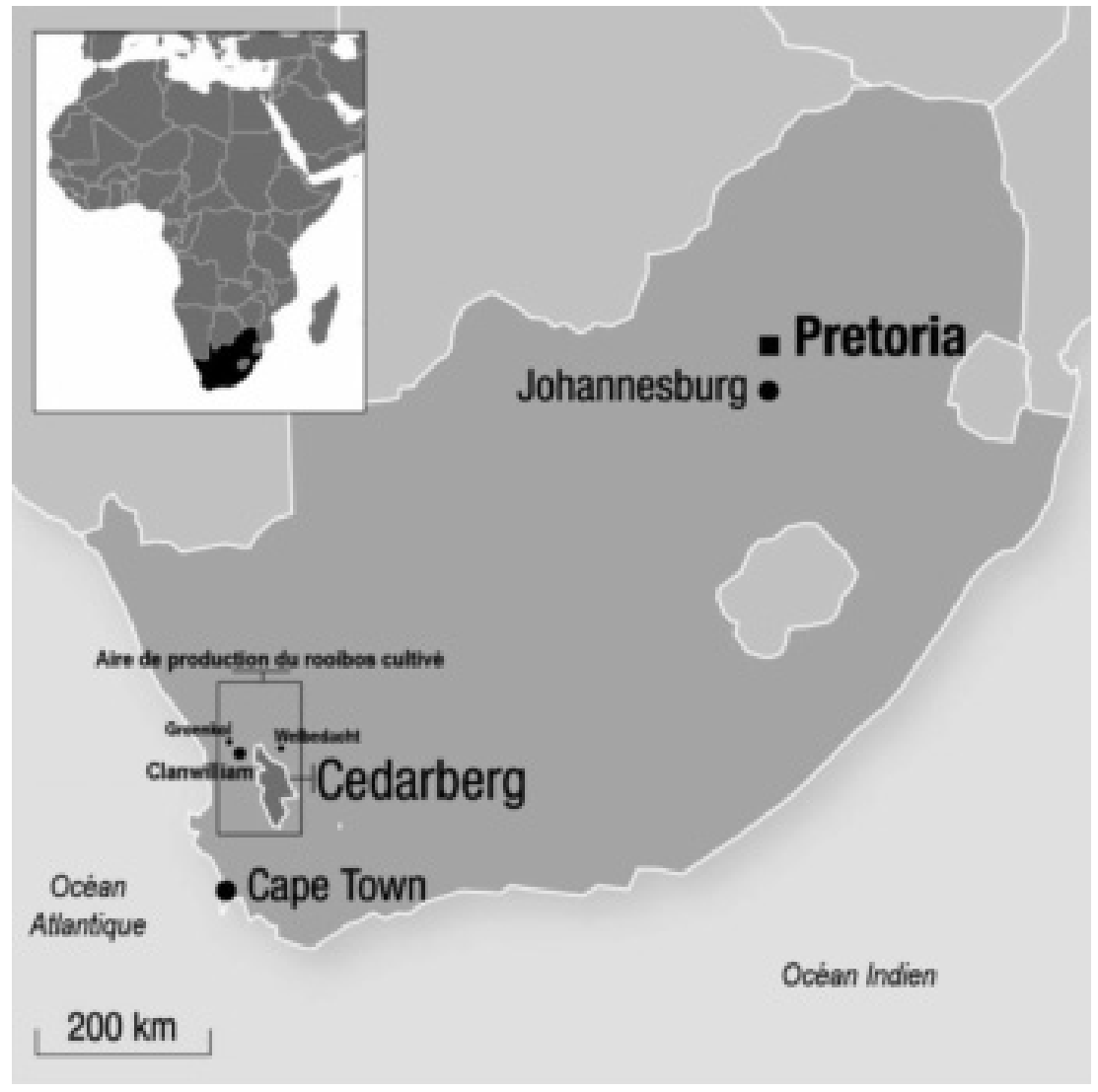

Carte 1-Localisation de l'aire de production du roibos

l'extérieur de cette zone, la mise en culture du rooibos est plus récente : les producteurs de la zone traditionnelle décrivent généralement ces nouvelles exploitations comme « opportunistes », profitant de l'attractivité du marché pour se lancer dans cette culture. De plus, acteurs locaux comme consommateurs nationaux et internationaux (à travers divers porte-paroles tels que les importateurs) reconnaissent également différentes sous-régions à l'intérieur de l'aire de production traditionnelle, dotées de réputations différentes. Certaines sont en effet connues pour produire un rooibos au goût supérieur, en lien direct avec des conditions agropédologiques spécifiques ${ }^{5}$.

5. Les conditions climatiques optimales à la croissance du rooibos sont une altitude d'au moins 500 mètres, un substrat sableux, et une pluviométrie comprise entre 350 et $650 \mathrm{~mm}$ par an [Dahlgren, 1968]. 


\section{Émergence d'un produit alimentaire spécifique : de la cueillette à la privatisation}

Quatre étapes clés du développement de la production de rooibos se dégagent des récits recueillis : la découverte de la plante tout d'abord, puis de sa valeur marchande ; sa mise en culture à grande échelle ; la découverte ensuite de nouvelles propriétés pharmaceutiques permettant de la promouvoir sur de nouveaux marchés ; enfin la dérégulation de la filière nationale qui participe du mouvement général de libéralisation des marchés opéré à la fin de l'apartheid. Le rooibos passe ainsi progressivement d'un produit de consommation locale, cueilli à l'état sauvage par les populations locales, à un produit de consommation internationale, à forte valeur ajoutée, cultivé à plus de $95 \%$ et occupant une place significative sur le marché des thés et tisanes. Il est consommé en particulier pour ses vertus médicinales.

C'est un marchand d'origine russe, Benjamin Ginsberg, qui perçoit la valeur marchande potentielle du rooibos au début du XXe siècle [Ginsberg, ibid.]. Il commence par acheter des sacs de rooibos aux descendants de familles khoekhoe ${ }^{6}$ [Ginsberg, ibid.] et les revend comme «thé des montagnes » en ville [South African Rooibos Council, ibid.]. Il fonde également la première marque de rooibos dans les années 1940, "11 o'clock», qui est encore aujourd'hui l'une des plus populaires en Afrique du Sud. C'est à la même époque que l'on voit apparaître les premières lois qui établissent les bases de la politique d'apartheid (telles le Native Land Act de 1913 qui interdit aux Africains de posséder des terres en dehors des « réserves » indigènes). La ségrégation raciale est cependant pratiquée depuis plusieurs siècles déjà, particulièrement dans la région du Cap. Elle est d'abord justifiée par des raisons économiques : les besoins de main d'œuvre sur les exploitations agricoles. À la fin du XVIII ${ }^{\mathrm{e}}$ siècle, la plupart des Khoekhoe de la colonie travaillent comme ouvriers agricoles pour les fermiers blancs, les Afrikaners, qui occupent les meilleures terres de la région [Penn, 2005].

Dans les années 1930, Benjamin Ginsberg incite un ami physicien à s'intéresser à la domestication de la plante. Après avoir testé plusieurs écotypes sauvages, il sélectionne la variété « rockland» [Van-Wyk, 2000], qui est encore aujourd'hui la seule cultivée. L'idée vient alors de promouvoir cette nouvelle culture peu coûteuse pour les agriculteurs de cette région aride ${ }^{7}$. Le rooibos est en effet un buisson bien adapté à son environnement, qui nécessite peu d'eau, d'intrants et de travail d'entretien. À cette époque cependant, la culture du rooibos reste limitée à l'initiative de quelques agriculteurs de la région de Clanwilliam qui approvisionnent l'espace sud-africain.

6. Ces derniers n'existent déjà plus en tant que groupe ethnique dans cette région au début du XX⿳亠口冋 siècle, décimés par les maladies et par les commandos organisés de colons [Fauvelle-Aymar, 2006]. Les survivants ont été embauchés comme ouvriers agricoles sur les fermes [Penn, 2005]. Leurs descendants sont inclus dans la catégorie «Coloured» (métis) pendant l'apartheid et sont aujourd'hui généralement considérés comme des «PDI» (Previously disadvantaged individuals). La plupart travaillent encore sur des fermes, le travail agricole étant l'une des rares sources de revenu dans cette région.

7. La variété « rockland» provient du nord-est de Clanwilliam, région très sèche où encore aujourd'hui, les principales productions agricoles sont le rooibos et les ovins. 
La pénurie de thé noir pendant la seconde guerre mondiale va provoquer un accroissement rapide de la production de rooibos [Morton, 1983] comme thé de substitution. L'effondrement du marché local après cette période faste motive les producteurs à fonder la première coopérative de production, la Clanwilliam Tea Cooperative en 1948, suivie de la fondation du Rooibos Tea Control Board (RTCB) en 1954 par le Ministère de l'Agriculture. Il s'agit d'un office public ayant l'exclusivité de la commercialisation du rooibos [Gress, 2004]. Cette époque correspond au renforcement de la politique d'apartheid avec l'accès au pouvoir du National Party (1948), qui est, entre autres, à l'origine de la loi sur la classification de la population (votée en 1950) et du retrait du droit de grève pour les travailleurs noirs (1953). L'autorité renforcée des agriculteurs blancs sur leurs ouvriers généralement décrite a posteriori comme « paternaliste » [Du Toit, 1992], le contrôle exclusif de l'État sur le marché qui laisse très peu de place aux initiatives locales des producteurs et la mise à l'écart de l'Afrique du Sud du commerce international ${ }^{8}$ expliquent sans doute la stagnation relative de la culture du rooibos.

Cette première phase de mise en culture et de mise sur le marché a permis d'institutionnaliser la filière et d'établir durablement la production, qui reste cependant faible. Les ventes sont encore limitées en Afrique du Sud (850 tonnes par an consommées à la fỉn des années 1950) et à l'étranger (14 tonnes consommées en 1968) [Morton, ibid.]. Le rooibos devient cependant peu à peu une boisson populaire en Afrique du Sud, au point d'être qualifié de «Boisson nationale sudafricaine » [Cheney et Scholtz, 1963]. C'est une action individuelle qui semble avoir provoqué un « déclic ». En 1968, une mère de famille de Pretoria popularise les propriétés anti-allergéniques du rooibos en particulier pour les enfants, participant ainsi à créer une nouvelle réputation pour ce produit. En 1971, elle fonde sa propre entreprise qui devient en quelques années l'une des plus grosses sociétés de cosmétiques à base de rooibos. Etablie à Pretoria, avec des filiales dans une dizaine de pays, cette entreprise dépose la marque « Rooibos » aux États-Unis en 1994.

La fin de l'apartheid provoque de nombreux bouleversements dans tous les domaines économiques et politiques, y compris dans l'industrie du rooibos. La fin des sanctions économiques sur les produits sud-africains, notamment, et la libéralisation de la filière suscitent l'entrée de nouveaux acteurs privés, en particulier dans la transformation et la commercialisation, permettant au marché du rooibos de se développer à l'international à partir des années 1990 [Erickson, ibid.]. L'organisme étatique, le RTCB, est démantelé et privatisé en 1993. Les producteurs fournisseurs du RTCB prennent possession de ses actifs et forment une société anonyme, Rooibos Ltd, dont ils sont les actionnaires. Il s'agit encore aujourd'hui de l'acteur le plus important du marché, avec environ $70 \%$ des parts

8. L'opinion internationale réagit dès les années 1960 après la répression policière dans le township de Sharpeville (événement connu comme le «massacre de Sharpeville ») et les premières sanctions économiques se font ressentir à partir des années 1970. 
de marché dont $95 \%$ environ du marché national [Snyman, 2007]. À partir de 1996, plusieurs compagnies privées de production et de transformation du rooibos sont créées, majoritairement tournées vers le marché de l'export [Gress, ibid.]. Par ailleurs, la réforme foncière, promise par l'African National Congress (ANC) lors de son arrivée au pouvoir en 1994, permet à certains producteurs préalablement désavantagés d'acquérir des terres. Si les résultats nationalement sont encore loin d'atteindre les objectifs visés ${ }^{9}$ [Anseeuw, 2004], deux coopératives de producteurs de rooibos coloured ont émergé de ce processus, avec l'appui d'ONG. Elles revendiquent un savoir-faire, celui de produire un rooibos de qualité différente, et ont rapidement développé des stratégies spécifiques de qualification tournées vers le commerce équitable et l'agriculture biologique.

Désormais réalisée à grande échelle, source fiable de revenu pour un plus grand nombre d'agriculteurs, prise en main par des industriels et appréciée des consommateurs, la production de rooibos prospère et devient un enjeu évident pour certains opérateurs économiques.

\section{Vers la reconnaissance de ce produit de terroir}

Du fait de ces spécificités tant culturelles que territoriales, médicinales et gustatives, le rooibos fait partie intégrante de l'héritage culturel sud-africain. Il est aussi populaire que le thé ou le café en Afrique du Sud ${ }^{10}$, et les populations et les paysages du Cedarberg, au Nord du Cap, sont clairement associés à la culture du rooibos. La dimension historique de ce produit, l'association de sa zone de production à la région du Cedarberg, son endémicité tout comme la spécificité des pratiques de culture, constituent autant de catégories distinctives que se sont appropriées certains acteurs locaux (producteurs), nationaux (organisation interprofessionnelle, distributeurs, institutions publiques) et internationaux (entreprises, consommateurs). Ces acteurs ont peu à peu contribué à faire du rooibos un produit de terroir selon la définition qu'en donnent L. Bérard, C. Delfosse et P. Marchenay $[1998 ; 2004]^{11}$. Souvent comparé à la vigne et au vin, le rooibos, s'il n'est pas désigné par les acteurs comme un «produit de terroir»- cette notion étant difficilement traduisible en anglais et sa vulgarisation dans le domaine agro-alimentaire étant un phénomène relativement récent en Europe [Bérard et Marchenay, 1995] -, est pensé comme un «produit typique sud-africain ». Il est intéressant

9. La réforme foncière mise en place par l'ANC (African National Congress) en 1994 prévoit de redistribuer $30 \%$ des terres en 5 ans. Dix ans après, seuls 1,5\% des 87 millions d'hectares de terres agricoles ont été redistribués.

10. Par exemple, le rooibos est très souvent présent dans les chambres d'hôtel et maisons d'hôtes en Afrique du Sud, à côté du thé et du café. De même, tous les restaurant et café proposent au moins un type de rooibos sur leur carte.

11. $\mathrm{Si}$ «L'expression "produit de terroir» (...) renvoie à une vaste catégorie fourre-tout», il est cependant possible de définir " un plus petit dénominateur commun », dont la définition " [croise] l'espace et le temps et repose sur des savoirs et des pratiques partagées » [Berard et Marchenay, 1998: 49-50]. Autrement dit, les produits de terroir sont des «produits agricoles et alimentaires inscrits en un lieu, dont la fabrication bénéficie d'une antériorité et relève de pratiques collectives. » [Berard, Delfosse et Marchenay, 2004 : 591] 
de souligner que le rooibos est un produit de terroir et non une production strictement identitaire. Il dépasse en effet les clivages ethniques, puisqu'il est aujourd' hui reconnu comme un produit associant des traditions d'usages provenant des Khoekhoe, des initiatives agricoles et commerciales essentiellement portées par des populations Afrikaners, et une contribution des populations coloured à son image et à sa reconnaissance internationale à travers le commerce équitable.

La construction d'une IG formaliserait la coexistence des trois éléments constitutifs de ce produit de terroir: une aire géographique délimitée, une trajectoire historique reconnue et des pratiques spécifiques de production et d'usage.

\section{Entre stratégies individuelles et construction collective de dispositifs}

De l'irruption de nouvelles préoccupations à l'émergence de dispositifs porteurs de nouvelles perspectives

\section{Demande de rooibos nationale et internationale depuis 1990}

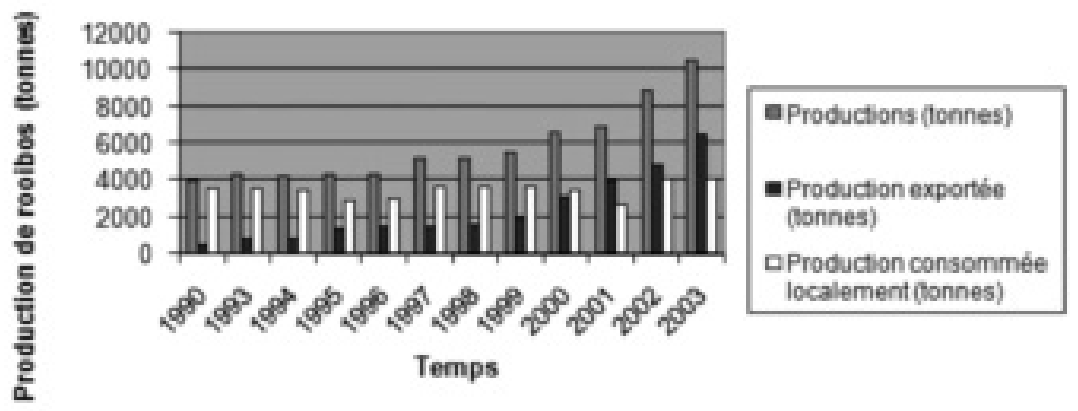

Figure 1 - La demande nationale de rooibos est stable depuis 1990, alors que la demande à l'export a augmenté (TISA, 2004)

La production de rooibos est multipliée par trois entre 1990 et 2003 (fig. 1). Avec cette augmentation, surtout liée au boom de l'export, l'aire de production est multipliée par deux, atteignant aujourd'hui environ 40000 hectares, et les pratiques s'intensifient [Hansen, 2006]. Du fait notamment de conditions climatiques propices, une production supérieure à la demande depuis 2005 entraîne une diminution significative du prix du rooibos (fig. 2). Ces variations d'offre et de prix sont associées à un nombre fluctuant de producteurs ; certains ne cultivent du rooibos que lorsqu'ils jugent le contexte économique favorable. Ceci génère de nouvelles inquiétudes et de nouveaux risques, perçus à des degrés divers par les acteurs de la filière : risques de dégradation de la qualité gustative liés à l'intensification des pratiques et aux comportements opportunistes de certains 
agriculteurs ; risques environnementaux et agronomiques liés à l'expansion de la culture hors de son aire de production traditionnelle; risques enfin d'usurpation et d'utilisation abusive du nom $«$ rooibos ${ }^{12}$.

\section{Production et prix du rooibos depuis 1990}
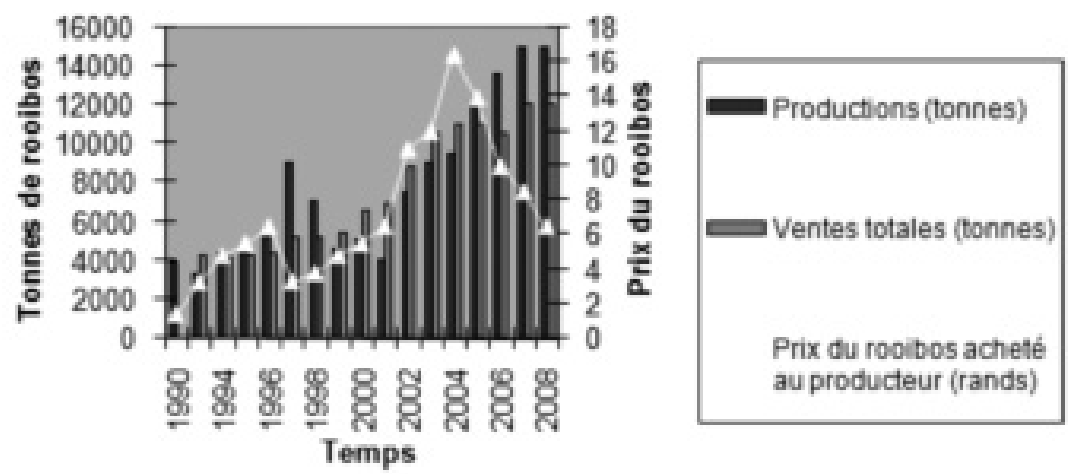

Figure 2 - Le prix du rooibos augmente lorsque la demande dépasse l'offre (de 1999 à 2005) et baisse lorsque l'offre dépasse la demande (tableau réalisé à partir des données communiquées par un producteur)

La vente de la marque déposée « rooibos » aux États-Unis par une entreprise sud-africaine a sa filiale américaine en $2001^{13}$, puis la volonté de cette filiale de faire valoir ses droits exclusifs sur cette marque, entraîne une longue bataille judiciaire entre cette dernière et l'industrie du rooibos, représentée par rooibos ltd. Le rooibos est finalement considéré par le bureau américain des brevets et des marques comme un terme générique en 2005 et l'enregistrement de la marque est annulé. Cette bataille, qui s'est avérée tres coûteuse pour la filire, a été fortement médiatisée en Afrique du Sud et a contribué largement à une prise de conscience collective de l'importance de protéger le patrimoine sud-africain. Au sein de la filière, cet événement a suscité une action collective structurée autour de la création du conseil sud africain du rooibos (CSAR) en avril 2005. En 2006, le CSAR initie un processus d'exploration du potentiel de développement d'une IG avec l'appui d'un projet de recherche action, le projet IPR DURAS ${ }^{14}$. Les échanges entre chercheurs et acteurs de la filière contribuent à une meilleure

12. Différents acteurs sud-africains impliqués dans la commercialisation du rooibos sur les marchés d'export font état de cas d'utilisation abusive de ce nom.

13. Il s'agit de l'entreprise de cosmétiques à base de rooibos, «Annique », qui déposa la marque « rooibos » en 1994 et à laquelle nous faisons référence dans la première partie.

14. Ce projet, intitulé «Linking farmers to markets through valorization of local resources : the case 
appréhension des enjeux collectifs et territoriaux liés au développement de la filière et du potentiel des IG pour y repondre. Un groupe de travail est constitué lors de l'assemblée générale du CSAR en 2006, par élection de représentants des différents types d'acteurs de la filière sud-africaine, c'est le «comité IG ».

Au-delà des risques d'usurpation du nom « rooibos » et d'une chute des prix, les producteurs confient d'autres inquiétudes : la difficulté de trouver de la main d'œuvre pour les opérations encore peu mécanisées (comme la récolte du rooibos); les incertitudes sur le foncier associées à la politique nationale de redistribution des terres. D'autres préoccupations, relayées cette fois par des organismes d'appui, sont directement traduites en projets. Des ONG, l'agence provinciale de conservation de la nature Cape Nature, des organismes de recherche ou encore le département de l'Agriculture du Western Cape, développent des partenariats avec le CSAR ou avec des groupes de producteurs sur des thèmes tels que la désertification (projet porté par l'ONG Environmental Monitoring Group) ou les prédateurs (projet The Cape Leopard Trust). Ces interventions d'origine extérieure à la filière contribuent à une prise de conscience collective des menaces qui pèsent sur le développement de la production du rooibos pour satisfaire la demande des marchés. Un projet intitulé «Sustainable Rooibos Initiative », porté au départ par Cape Nature en liaison avec des sociétés de conservation de la nature, vise à développer des outils de conservation pour la filière du rooibos. Interpellé par Cape Nature et plus généralement par la société civile et les pouvoirs publics sur ces questions, le CSAR s'est engagé dans cette perspective et contribue maintenant à financer le projet conjointement avec Cape Nature [Cape Nature, 2008]. Cette initiative et l'engagement du CSAR sont en fait inspirés par une initiative antérieure, portée en grande partie par des sociétés de conservation de la nature, dans le secteur du vin [Allix, 2009], et dont le succès a largement été médiatisé.

L'ambition de gérer collectivement la biodiversité est donc indépendante au départ de la démarche de construction d'une IG au sein du CSAR. Cependant, l'approche de l'initiative biodiversité est comparable sur certains plans à la démarche IG. En effet, cette démarche repose sur le volontariat des agriculteurs et met l'accent sur la possibilité de labelliser les exploitations qui adoptent des comportements favorables à la conservation de la biodiversité (mise en défens de certaines parties de l'exploitation par exemple). De plus, si son origine, comme celle de l'IG est exogène à la filière, elle répond à des menaces ressenties par les acteurs.

En ouvrant ainsi une nouvelle perspective de qualification collective des pratiques, les dispositifs de construction d'une IG et de conservation de la biodiversité s'imposent comme des cadres normatifs pour négocier, au sein de la filière, la

for intellectual property rights of indigenous resources » et financé par le Ministère Français des Affaires Étrangères et Européennes, est coordonné par l'Université de Pretoria, avec l'appui du CIRAD et du Département de l'Agriculture du Western Cape. Il vise à explorer le potentiel des IG en Afrique Australe comme outil d'accès aux marchés des petits producteurs. 
définition de bonnes pratiques agricoles et de stratégies commerciales cohérentes avec leurs principes.

\section{Des stratégies individuelles variées pour valoriser le rooibos}

Cependant, si la plupart des opérateurs politiques et économiques sont conscients que la «mise en patrimoine » du rooibos associée à la démarche IG est un processus en construction, on n'observe pas encore de réelle communauté de pratiques. Si les éléments fondateurs d'une représentation commune du rooibos en tant que produit « local » ou «typique » sont bien identifiés, ni les itinéraires techniques envisagés pour l'obtenir ni les pratiques commerciales ne sont homogènes. Chacun maîtrise et revendique un savoir ou des intérêts spécifiques. La biodiversité, quant à elle, n'est encore présente qu'implicitement dans les pratiques, comme un élément passif du contexte. Les deux trajectoires individuelles de producteurs décrites ci-dessous (encadrés cas 1 et cas 2) illustrent ces pratiques différentes de production et de valorisation d'une part, et l'importance implicite de la biodiversité d'autre part ${ }^{15}$. Elles permettent de mieux comprendre le contexte et les contenus possibles des négociations autour de la construction d'une IG sur le rooibos.

On a ici deux stratégies contrastées pour valoriser un rooibos et le différencier des autres sur les marchés. L'une est basée sur les qualités organoleptiques et l'importance du réseau de clientèle, l'autre s'appuie sur un leadership technique et sur l'efficacité de ses innovations pour produire une qualité maîtrisée. Elles ont cependant en commun de revendiquer une qualité particulière, justifiant la création d'une marque individuelle. Traduction d'une réputation gustative et géographique ou d'un style technique qui justifie l'évocation d'un ancrage territorial [MoityMaïzi, à paraître], la marque de chacune est associée sur les emballages à une iconographie distinctive. Il y est fait référence à la flore et à la faune emblématiques des paysages sud-africains pour le consommateur étranger, alors même que ces producteurs ne s'inscrivent pas explicitement dans une démarche de défense de la biodiversité. Celle-ci semble intervenir comme symbole venu en renfort du nom et d'une réputation. Pourtant, c'est bien une connaissance complexe du milieu (sols, altitude, pluviométrie, diversité végétale) qui fonde leurs choix techniques (dans la sélection d'une zone de défriche pour implanter la culture en particulier) et la diversité des pratiques. La biodiversité est donc présente sans être explicitement envisagée comme un enjeu dans leurs pratiques.

15. Il est important de préciser que les deux cas exposés concernent des producteurs ayant développé leur propre marque, lesquels sont peu nombreux dans la filière mais en forte augmentation ces dix dernières années. 


\section{Cas 1 : Biedouw Valley (fig. 3)}

La famille Van der Merwe a acheté la ferme «Welbedacht » dans la région de Biedouw Valley en 1996. Initialement producteurs de légumes dans cette région très isolée, ils se sont tournés vers la production de rooibos depuis une dizaine d'années. Leurs champs sont situés à une quinzaine de kilomètres de la ferme, sur un plateau situé entre 900 et 1000 mètres d'altitude [Biedouw Valley Rooibos, 2008]. Le rooibos cultivé dans cette zone bénéficie d'une forte réputation. Son goût serait particulier et sa qualité supérieure grâce aux caractéristiques agro-pédologiques de cette région. C'est ce qui a décidé la famille Van der Merwe à déposer sa propre marque. Cette décision est d'abord motivée par une demande des touristes de passage, puis par l'ambition de se distinguer de l'industrie du rooibos en valorisant mieux leur propre production qui était jusque là mélangée avec celles d'autres producteurs, parfois de qualité inférieure. La marque «Biedouw Valley» a donc été créée en 1997 et la famille Van der Merwe vend aujourd'hui surtout à l'export. Le discours de ces acteurs sur la qualification de leur produit ne se réfère pas à des pratiques spécifiques, ni à une éventuelle antériorité sur le territoire, mais plutôt à l'intérêt et à la confiance des consommateurs ${ }^{16}$, établis dans une relation marchande de proximité et favorisés par le caractère touristique de la région. La vallée de Biedouw est en effet une des régions du Cedarberg connue pour ses plantes endémiques rares qui fleurissent au printemps, ses formations rocheuses spectaculaires et de nombreuses peintures rupestres des Bushmen [Slingsby et Coombe, 2001], ce qui en fait une destination touristique particulièrement réputée au niveau national et international.

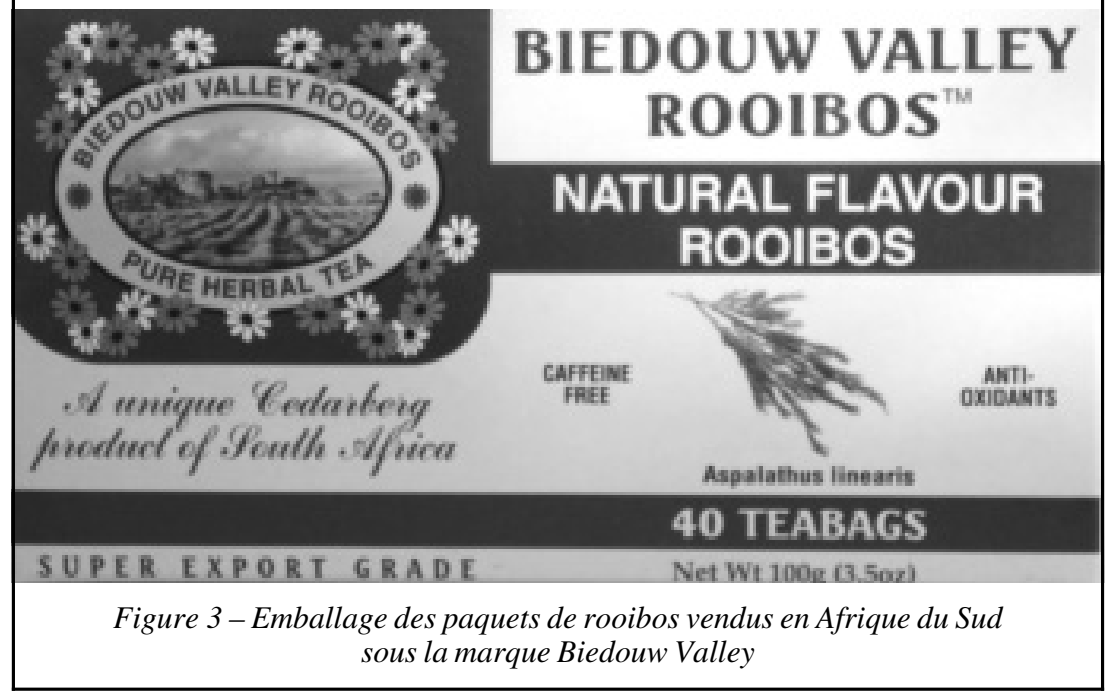

16. Par exemple, les mails reçus par des consommateurs du monde entier sont précieusement conservés dans un classeur, et ont largement été cités lors de l'entretien mené avec une personne de cette exploitation. 


\section{Cas 2 : African Dawn (fig. 4)}

La famille Engelbrecht a commencé à produire du rooibos dans les années 1950. Installée dans l'une des zones de production les plus réputées, c'est l'une des familles les plus anciennes dans la production de rooibos ${ }^{17}$. Elle est propriétaire de plusieurs fermes, dont la plus importante, Groenkol, abrite une usine de transformation, de stérilisation et d'emballage. L'exploitation est transmise et gérée depuis trois générations.

Les hommes de cette famille se sont très tôt distingués par des techniques de mécanisation innovantes. Oubaas Engelbrecht se présente, et est reconnu, comme l'inventeur de la machine à récolter le rooibos. Il justifie l'efficacité de cet outil et la qualité finale de son rooibos, face à ses détracteurs ${ }^{18}$, par une plus grande rapidité d'action, par une meilleure hygiène (les tiges de rooibos coupées sont directement stockées sur une remorque et ne touchent plus le sol) et par l'homogénéité de la récolte. Cette maîtrise technologique lui a valu un certain nombre de prix, qui ornent les locaux de l'entreprise. Forte de ces symboles et de sa réputation technicienne, la famille Engelbrecht est aussi l'une des plus grosses productrices ${ }^{19}$, ce qui l'a incitée à créer une marque (African Dawn) et une entreprise de transformation du rooibos (Big Five Company) en 1998, après la dérégulation de l'industrie. Du fait de sa capacité de production et de la reconnaissance dont elle bénéficie, cette famille a fait le choix de ne commercialiser sous sa marque que son propre rooibos, important le concept de «domaine » utilisé dans l'industrie du vin.

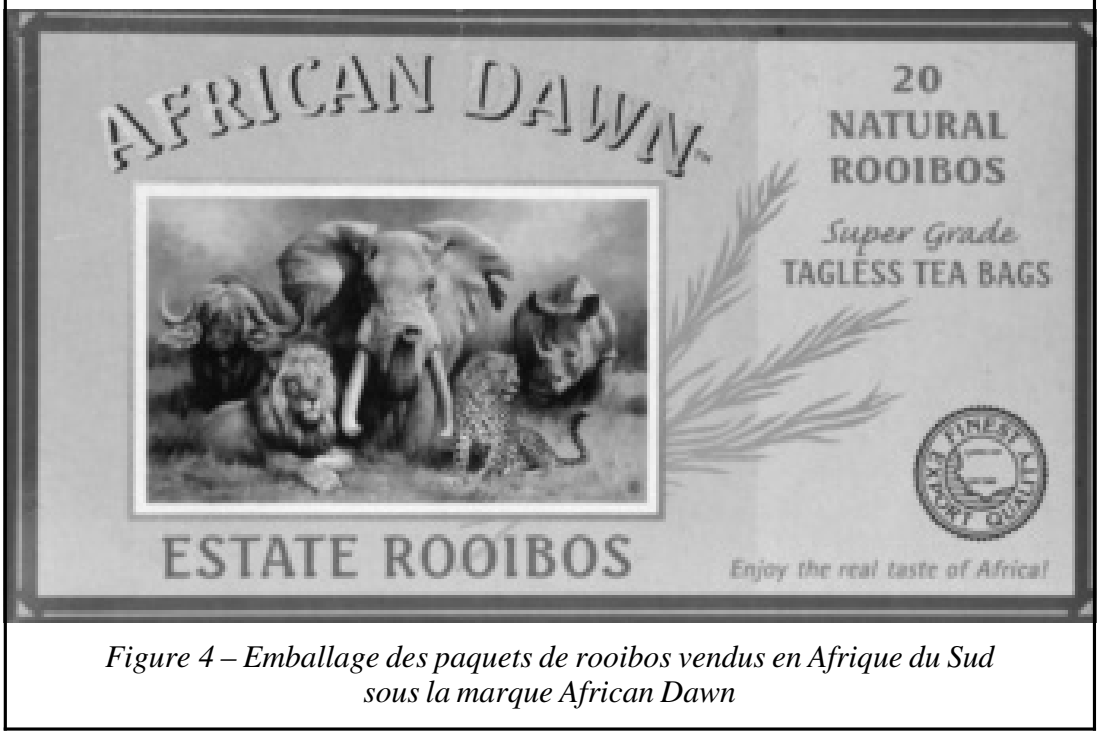

17. C'est ainsi qu'ils se présentent et sont généralement reconnus par les autres producteurs de rooibos.

18. Beaucoup de producteurs qui récoltent le rooibos à la main le justifient d'abord par une volonté de préserver la qualité : les machines couperaient les plants de rooibos à la même hauteur, tandis que le travail à la main permet d'ajuster la coupe.

19. Avec une production d'environ 350 tonnes de rooibos par an, elle figure parmi les 10 plus gros producteurs. 


\section{La construction collective de I'lG : fondements d'un premier réseau}

La culture intensive de rooibos qualifiée d' «opportuniste» participe de la diversité des pratiques qui vient d'être illustrée. Cependant, bien que n'étant pas proscrite, elle relève de choix techniques individuels porteurs de risques de plus en plus reconnus et motive la vision partagée d'une menace sur la biodiversité au sein du comité IG, élu comme porte-parole de la diversité des pratiques. Ce comité regroupe un agriculteur d'exploitation commerciale de grande taille qui valorise directement sa production (cf. le cas 2 ci dessus); le directeur de la plus grosse entreprise de transformation (Rooibos Ltd), qui est également le plus grand producteur et Président du Bureau du CSAR ; un transformateur qui siège aussi au Bureau du CSAR ; un petit producteur d'une des deux coopératives commercialisant le rooibos sous les labels Agriculture Biologique et Commerce Équitable et un représentant des ONG partenaires de ces coopératives. Y sont associés par ailleurs deux partenaires institutionnels du projet : un chercheur du département de l'agriculture du Western Cape et un chercheur du CIRAD basé à l'université de Pretoria.

Ce comité réunit ainsi des acteurs aux trajectoires économiques et politiques différentes. Ces acteurs s'inscrivent dans différents registres discursifs et normatifs qui se sont avérés sources de tension dans d'autres contextes. En particulier, le représentant des ONG se pose comme porte-parole du groupe des petits producteurs historiquement non reconnus dans la filière. Il joue un rôle actif dans la dénonciation des stratégies des «grands exploitants » pour intégrer les circuits du commerce équitable. En effet, cette intégration est considérée par les petits producteurs comme une concurrence déloyale du fait des disparités entre leurs capacités de production et celles des grands exploitants, et de la perspective d'une baisse du différentiel de prix dont ils bénéficient pour l'instant.

De leur côté, les partenaires du projet IPR DURAS associés au comité jouent un rôle dans le processus de «traduction » du concept d'IG et de ses contenus pour la filière sud africaine. Tout en essayant de renseigner les débats dans le comité et de donner à voir, dans la mesure du possible, les implications de différents choix, ils ne se posent pas en arbitres des stratégies économiques dans la mise en place de l'IG.

\section{Un dispositif propice aux interactions entre IG et biodiversité}

Tout en admettant l'existence de différentes pratiques, les membres du Comité voient dans l'IG un moyen de faire converger leurs intérêts et de répondre à un certain nombre d'inquiétudes, autour de la construction d'un dispositif de protection du nom et de qualification du produit. Initialement, ce collectif se concentre sur deux enjeux économiques : la gestion et la promotion de la réputation du nom rooibos et de ses qualités. Face aux risques d'une «internationalisation» de la filière, la construction de l'IG porte sur la protection du nom « rooibos » qui est commun à tous les acteurs de la filière. Elle est donc envisagée dans une démarche 
nécessairement inclusive, intégrant a priori tous les producteurs, quelle que soit leur localisation en Afrique du Sud, et prenant même en considération de nouvelles zones potentielles de production dans la région pour tenir compte de l'expansion en cours de la filière. Le comité envisage donc la délimitation d'un vaste territoire qui intègre toute l'aire de production, sans distinguer la zone réputée pour son rooibos de qualité supérieure de l'aire d'expansion de cette culture. Dans cette logique intégratrice, la codification des pratiques consiste principalement à définir un niveau minimal de qualité que les producteurs doivent atteindre [Biénabe et al., à paraître]. Si cette logique permet de répondre à des enjeux de protection et de réputation collective, elle ne facilite pas le débat sur les questions posées par la diversité croissante des pratiques et des points de vue sur la qualité du rooibos notamment.

La biodiversité ne s'inscrit pas d'emblée au cœur de ces débats. Cependant, très rapidement, les réflexions du comité l'amènent à identifier des liens entre la construction de l'IG, fondée sur la qualification des pratiques et du produit, et la démarche de conservation de la biodiversité. L'endémicité du rooibos dans un milieu particulier et fragile, le fynbos, est en effet reconnue comme un élément fondateur de la spécificité du rooibos ; et la protection de ce milieu est considérée comme déterminante dans une stratégie collective de gestion durable du rooibos. La biodiversité émerge donc comme un enjeu d'avenir, collectivement admis, au sein de la démarche de construction de l'IG.

Un consultant de Cape Nature est alors invité à rejoindre le comité. Depuis son intégration, la biodiversité participe ouvertement au processus de construction de l'IG, le consultant jouant au départ le rôle de traducteur des enjeux de biodiversité en lignes d'action possibles (maintien ou création de corridors, gestion des zones humides, gestion de la faune sauvage...). Celles-ci sont examinées par le comité sous le prisme de leur pertinence vis-à-vis des pratiques de production et des enjeux propres à la filière. À partir d'une vision systémique portée par le consultant, la biodiversité devient, dans ce collectif et ses débats, un référent pour raisonner et qualifier les pratiques. Les discussions conduisent ainsi à reconnaître l'existence de différents modes d'exploitation du milieu qui ont des impacts différents sur la biodiversité, et de ce fait doivent faire l'objet de traitements différents dans le futur cahier des charges de l'IG. Une distinction est par exemple opérée entre exploitations en fonction de l'intensité de la culture. Ainsi, si le principe de l'établissement de corridors biologiques au sein des exploitations est retenu, l'obligation d'inclure entre les champs de rooibos des bandes de terre en végétation naturelle ne serait appliquée qu'aux fermes comptant plus de $50 \%$ de terres cultivées. Cette distinction constitue de fait une reconnaissance des différences entre zone traditionnelle de production extensive, dans laquelle les terres sont faiblement valorisées sur le plan agricole, et zone d'expansion beaucoup plus intensive. La biodiversité « agit » dans le comité en enrichissant et en guidant les choix dans les procédures de codification des pratiques.

Aujourd'hui, la construction de l'IG semble concerner au moins autant les éléments d'une réputation fondée sur l'origine de l'usage, la spécificité de la plante 
et les savoirs qui y sont associés, que les techniques ou procédés de culture et de transformation; elle intègre des critères et des questions nouvelles liés à la biodiversité. L'originalité de la démarche IG pour le rooibos repose sur le fait qu'elle a intégré la conservation de la biodiversité comme un élément du processus de qualification, suscitant une réflexion collective sur les pratiques individuelles de production. L'idéal initial que constitue la «conservation de la biodiversité » contribue maintenant aux négociations pour orienter les choix. L'ambition de gérer collectivement la biodiversité n'apparaît donc plus comme un simple slogan, ni comme un idéal politique ou moral dont on ne maîtriserait guère les traductions concrètes, mais participe activement de la constitution d'un véritable dispositif (au sens que lui donnait Foucault), reliant une grande diversité d'acteurs (individuels et collectifs, humains et non humains) autour d'un projet commun de raisonnement et de qualification des pratiques. La démarche de construction de l'IG fournit le cadre de ce dispositif collectif. Dans ses « missions » de porte-parole et de leader, ralliant diverses « voix » autour d'enjeux communs, le comité constitue au final un espace de médiation entre plusieurs « mondes » (les petits et grands producteurs, les acteurs de la filière et les institutions publiques parmi lesquelles des organismes de recherche et des acteurs de la conservation). Il se montre apte à faire émerger un consensus sur la définition des contours et conditions d'une future IG pour le rooibos, mettant en cohérence des pratiques individuelles et différents enjeux collectifs.

\section{Conclusion}

Le processus de qualification du rooibos que nous venons de décrire évoque au fond un tournant souligné par B. Latour : "partout où l'on avait modernisé, il faut maintenant écologiser » [Latour, 1995: 26]. De la domestication de la plante à sa commercialisation sur de multiples marchés, rendue possible par une mécanisation partielle et une intensification de la production, la «modernisation » couvre en effet, au moins de manière métaphorique, ces évolutions convoquant de nouveaux acteurs pour repenser le développement de la filière rooibos. Dans cette «écologisation », on peut distinguer deux temps forts. L'IG apparaît d'abord comme instrument et signe distinctif au service de l'économie de la filière ayant vocation de protection du nom et de la réputation d'un produit de terroir. Elle ne sanctionne alors ni la culture intensive d'une plante, ni l'expansion des aires de culture, mais reconnaît déjà la biodiversité comme un élément fondateur de la spécificité du rooibos. Dans un second temps, la construction de l'IG consiste à raisonner les pratiques en puisant cette fois dans le registre normatif de la conservation de la biodiversité des arguments qui intègrent l'évaluation des menaces et les connaissances des producteurs localisés dans divers milieux.

Porteur de ce projet de construction d'une IG désormais associée à une logique conservatoire de la biodiversité, le comité défend une démarche originale permettant de valoriser la biodiversité dans l'image finale du produit et d'intégrer la 
reconnaissance de pratiques respectueuses de la biodiversité dans le futur cahier des charges [Biénabe et al., 2008]. Enfin, il s'affirme aujourd'hui comme un leader pour la filière et pour toute l'Afrique du Sud (à travers le CSAR) en faisant pression pour qu'un cadre national légal soit créé pour la mise en place d'IG en Afrique du Sud.

Cependant, de nombreuses questions subsistent. Au-delà de l'espace du comité, la conservation de la biodiversité constituera-t-elle un élément véritablement fédérateur entre tous les producteurs ? Ses capacités de mise en débats et d'arbitrage seront-elles à même de produire une codification des pratiques dans laquelle se reconnaîtront les producteurs et, sans plus d'intervention de l'État, de constituer ensuite une situation exemplaire sur laquelle s'appuieraient d'autres filières pour mettre en place des IG ? À ce stade du processus d'émergence des IG en Afrique en Sud, ces questions restent ouvertes.

\section{BibLIOGRAPHIE}

Allix G. [2009], «Les viticulteurs du Cap font leur révolution verte », Le Monde, 9 janvier 2009: 4.

ANSEEUW W. [2004], «La réforme foncière en Afrique du Sud : des résultats peu convaincants », in Guillaume P., et al. (éd.), L'Afrique du Sud 10 ans après. Transition accomplie?, IFAS-Karthala, p. 129-148.

Bérard L., Marchenay P. [1995], «Lieux, temps et preuves. La construction sociale des produits de terroir », Terrain, 24, p. 153-164.

Bérard L., Marchenay P. [1998], « Patrimoine et modernité : les produits du terroir sous les feux de la rampe », Journal des Anthropologues, 74, p. 47-66.

Bérard L., Delfosse C. et Marchenay P. [2004], «Les "produits de terroir" : de la recherche à l'expertise », Ethnologie Française, 34, p. 591-600.

Biedouw Valley Rooibos [2008], Consulté le 28 août 2008, http://www.biedouwtea.com/.

BiÉnABe E., Bramley C., Kirsten J. [A paraitre] « An economic analysis of the evolution in intellectual property strategies in the South African agricultural sector : the use of intellectual property in the rooibos industry ». Chapitre d'ouvrage édité par l'OMPI.

Biénabe E., Bramley C., Troskie D., Kirsten J. [2008], « The move towards geographical indication protection within the rooibos industry : a case in point for harnessing geographical indication potential in South Africa », Communication au séminaire final SINER-GI, Genève, 23, 24 juin 2008.

CAelers D. [2007], «Local rooibos tea researchers brew up success », Cape Argus, 1.

Cape Nature [2008], consulté le 17 juillet 2008, http://www.capenature.org.za/about.htm? $\operatorname{sm}[\mathrm{p} 1][$ category $]=582$.

Cheney R. H. et Scholtz E. [1963], « Rooibos Tea, A South African Contribution to World Beverages », Economic Botany, 17, p. 186-194.

DAhlgRen R. [1968], «Revision of the genus Aspalathus. II. The species with ericoid and pinoid leaflets. Subgenus Nortieria. With remarks on rooibos tea cultivation », Bot. Notiser, 121, p. 165-208. 
Du TolT A. [1992], «The Farm as Family. Paternalism, Management and Modernisation on Western Cape Wine and Fruit Farms », Stellenbosch, Center for Rural Legal Studies : 58.

ERICKSON L. [2003], « Health Benefits of Rooibos. Red Bush Tea from South Africa », HerbalGram, The Journal of the American Botanical Council, 59, p. 34-45.

Fauvelle-Aymar F.-X. [2006], Histoire de l'Afrique du Sud, Paris, Editions du Seuil.

Ginsberg B. [1976], « Rooibos Tea », The Herbal Review, p. 7-12.

Gress T. [2004], South African Rooibos Industry. Opportunities and Constraints With Special References To the German Consumer Market, Thèse de Master Business Science, University of Cape Town.

Hansen T. [2006], A Sustainable production strategy for the South African Rooibos Tea Industry, Rapport commissionné par Cape Nature et le CSAR, Cape Town.

Latour B. [1995], « Moderniser ou écologiser ? À la recherche de la "septième" cité », Écologie politique : science, culture, société, 3 : 5-27.

Olivier DE SARDAN J.P. [1995], La politique du terrain. Sur la production de données en Anthropologie, Èd Parenthèses, Paris : 71-109.

MoITY-Maïzi P. [à paraître], «L'ancrage territorial mis en question », in J. Muchink et C. de Sainte Marie (dir.) ouvrage de synthèse du programme PIDAL, INRA.

Morton J.F. [1983], «Rooibos Tea, Aspalathus linearis, a Caffeineless Low-Tannin Beverage », Economic Botany 37 (2) : 164-173.

Penn N. [2005], The forgotten Frontier. Colonist and Khoisan on the Cape's Northern Frontier in the 18th Century. Cape Town, Double Storey Book.

Rooibos Limited [2008], consulté le 17 juillet 2008, http://www.rooibosltd.co.za

Slingsby P., Coombe E. [2001], Beyond the Cederberg, Agter-Pakhuis, Biedouw, Wupperthal, Cape Town, Paperback.

Snyman S. [2007], Subsector study : Rooibos Tea. Rapport commissioné par le National Agricultural Marketing Council, Pretoria.

South African Rooibos Council [2008], consulté le 10 juillet 2008, http://sarooibos.co.za/

TISA [2004], CSP Development for Rooibos Sector, rapport commissionné par Trade and Investment South Africa, Pretoria.

Van-Wyk B.-E. et al. [2000], Medicinal Plants of South Africa, Pretoria, Arcadia.

WTO [2008], consulté le 14 août 2008, http://www.wto.org/english/tratop_e/trips_e/trips_e.htm 